Proyecciones Journal of Mathematics

Vol. 41, $\mathrm{N}^{o}$ 1, pp. 163-176, February 2022.

Universidad Católica del Norte

Antofagasta - Chile

\title{
Regularity of the extensions of a double fuzzy topological space
}

\author{
S. Vivek \\ St. Thomas College, India \\ and \\ Sunil C. Mathew \\ Deva Matha College Kuravilangad, India \\ Received: August 2020. Accepted : August 2021
}

\begin{abstract}
This paper studies the regularity of the extensions of a double fuzzy topological space. The interrelations among certain families of closed sets in a double fuzzy topological space and its extensions are also investigated.
\end{abstract}

Keywords: Double fuzzy topology; Closed set; Regular space

2010 Mathematics Subject Classification: $54 A 40$. 


\section{Introduction}

The study on the extensions of a double fuzzy topological space was initiated in [8] and the authors examined whether the connectedness and compactness of a given double fuzzy topological space are carried over to its extensions. Further, in [11] it is proved that the extensions need not preserve the normality of a double fuzzy topological space and obtained some conditions which ensure the normality of the extended space. Recently, some interrelations among the families of $(r, s)$-regular fuzzy closed sets in a double fuzzy topological space and its extensions were obtained in $[9,12]$. Also, some studies on mixed fuzzy topological spaces can be seen in $[7,13,14,15,16]$.

In this paper, we inquire the regularity of the extension of a regular double fuzzy topological space. Though the extensions do not preserve regularity in general, some conditions which ensure the regularity of the extended space are obtained. Moreover, certain families of closed sets in a double fuzzy topological space and its extensions are investigated and some types of extensions under which these families remain unchanged are identified.

\section{Preliminaries}

Throughout this paper $X$ stands for a non-empty set and some particular sets are identified as, $I=[0,1], I_{0}=(0,1], I_{1}=[0,1)$ and $I_{0} \oplus I_{1}=\{(r, s) \in$ $\left.I_{0} \times I_{1}: r+s \leq 1\right\}$.

Definition 2.1. [5] Let $\left(\tau, \tau^{*}\right)$ be a pair of functions from $I^{X}$ to $I$ satisfying

(i) $\tau(f)+\tau^{*}(f) \leq 1, \forall f \in I^{X}$,

(ii) $\tau(\underline{0})=\tau(\underline{1})=1, \tau^{*}(\underline{0})=\tau^{*}(\underline{1})=0$,

(iii) $\tau\left(f_{1} \wedge f_{2}\right) \geq \tau\left(f_{1}\right) \wedge \tau\left(f_{2}\right)$ and $\tau^{*}\left(f_{1} \wedge f_{2}\right) \leq \tau^{*}\left(f_{1}\right) \vee \tau^{*}\left(f_{2}\right), f_{i} \in$ $I^{X}, i=1,2$,

(iv) $\tau\left(\bigvee_{i \in \Delta} f_{i}\right) \geq \bigwedge_{i \in \Delta} \tau\left(f_{i}\right)$ and $\tau^{*}\left(\bigvee_{i \in \Delta} f_{i}\right) \leq \bigvee_{i \in \Delta} \tau^{*}\left(f_{i}\right), f_{i} \in I^{X}, i \in \Delta$

Then the pair $\left(\tau, \tau^{*}\right)$ is called a double fuzzy topology on $X$ and the triplet $\left(X, \tau, \tau^{*}\right)$ is called a double fuzzy topological space or dfts in short. 
Definition 2.2. [3] Let $\left(X, \tau, \tau^{*}\right)$ be a dfts. For $(r, s) \in I_{0} \oplus I_{1}$, a fuzzy set $f$ is called

(i) $(r, s)$-fuzzy open if $\tau(f) \geq r$ and $\tau^{*}(f) \leq s$ and

(ii) $(r, s)$-fuzzy closed if $f^{c}$ is $(r, s)$-fuzzy open.

The collections of all $(r, s)$-fuzzy open sets and $(r, s)$-fuzzy closed sets are respectively denoted by $\mathcal{O}_{\tau, r, s}$ and $\mathcal{C}_{\tau, r, s}$.

Definition 2.3. [3] Let $\left(X, \tau, \tau^{*}\right)$ be a dfts. For each $(r, s) \in I_{0} \oplus I_{1}$, $f \in I^{X}$ the operator $C_{\tau, \tau^{*}}: I^{X} \times I \times I \rightarrow I^{X}$ defined by

$$
C_{\tau, \tau^{*}}(f, r, s)=\bigwedge\left\{g \in I^{X} \mid f \leq g, \tau\left(g^{c}\right) \geq r, \tau^{*}\left(g^{c}\right) \leq s\right\}
$$

is called the double fuzzy closure operator on $\left(X, \tau, \tau^{*}\right)$.

Definition 2.4. [3] Let $\left(X, \tau, \tau^{*}\right)$ be a dfts. For each $(r, s) \in I_{0} \oplus I_{1}, f \in I^{X}$ the operator $I_{\tau, \tau^{*}}: I^{X} \times I \times I \rightarrow I^{X}$ defined by

$$
I_{\tau, \tau^{*}}(f, r, s)=\bigvee\left\{g \in I^{X} \mid f \geq g, \tau(g) \geq r, \tau^{*}(g) \leq s\right\}
$$

is called the double fuzzy interior operator on $\left(X, \tau, \tau^{*}\right)$.

Definition 2.5. [6] Let $\left(X, \tau, \tau^{*}\right)$ be a dfts, $f \in I^{X},(r, s) \in I \oplus I$. Then

1. $f$ is called $(r, s)$-regular fuzzy open (or $(r, s)$-rfo) if $f=I_{\tau, \tau^{*}}\left(C_{\tau, \tau^{*}}(f, r, s), r, s\right)$.

2. $f$ is called $(r, s)$-regular fuzzy closed (or $(r, s)$-rfc) if $f=C_{\tau, \tau^{*}}\left(I_{\tau, \tau^{*}}(f, r, s), r, s\right)$.

We denote the collection of all $(r, s)$-rfc sets by $R \mathcal{C}_{\tau, r, s}$ and the collection of all $(r, s)$-rfo sets by $R \mathcal{O}_{\tau, r, s}$.

Abbas [1] introduced the concept of $(r, s)$-generalized fuzzy closed sets in a dfts as the following:

Definition 2.6. [1] Let $\left(X, \tau, \tau^{*}\right)$ be a dfts, $f, h \in I^{X},(r, s) \in I_{0} \oplus I_{1}$, then $f$ is called

1. ( $r, s)$-generalized fuzzy closed (for short, $(r, s)$-gfc) set if $C_{\tau, \tau^{*}}(f, r, s) \leq$ $h$ whenever $f \leq h$ and $h \in \mathcal{O}_{\tau, r, s}$. 
2. $(r, s)$-generalized fuzzy open (for short, $(r, s)$-gfo) set if $f^{c}$ is a $(r, s)$ gfc set.

We denote the collection of all $(r, s)$-gfc sets by $G \mathcal{C}_{\tau, r, s}$ and that of $(r, s)$-gfo sets by $G \mathcal{O}_{\tau, r, s}$.

The $(r, s)$-regular generalized fuzzy open sets and $(r, s)$-regular generalized fuzzy closed sets were introduced by Ghareeb in [2] as follows:

Definition 2.7. [2] Let $\left(X, \tau, \tau^{*}\right)$ be a dfts, $f, h \in I^{X},(r, s) \in I_{0} \oplus I_{1}$, then $f$ is called

1. $(r, s)$-regular generalized fuzzy closed (for short, $(r, s)$-rgfc) set if $C_{\tau, \tau^{*}}(f, r, s) \leq h$ whenever $f \leq h$ and $h \in R \mathcal{O}_{\tau, r, s}$.

2. $(r, s)$-regular generalized fuzzy open (for short, $(r, s)$-rgfo) set if $f^{c}$ is a $(r, s)$-gfc set.

We denote the collection of all $(r, s)$-rgfc sets by $R G \mathcal{C}_{\tau, r, s}$ and the collection of all $(r, s)$-rgfo sets by $R G \mathcal{O}_{\tau, r, s}$.

In [4], the authors introduced the concepts of $(r, s)$-fuzzy $b$-closed sets and $(r, s)$-fuzzy $b$-open sets in a dfts.

Definition 2.8. [4] Let $\left(X, \tau, \tau^{*}\right)$ be a dfts. A fuzzy set $f$ is called

1. $(r, s)$-fuzzy b-closed (briefly, $(r, s)$-fbc) if

$$
\left(I_{\tau, \tau^{*}}\left(C_{\tau, \tau^{*}}(f, r, s), r, s\right)\right) \wedge\left(C_{\tau, \tau^{*}}\left(I_{\tau, \tau^{*}}(f, r, s), r, s\right)\right) \leq f
$$

2. $(r, s)$-fuzzy b-open (briefly, $(r, s)$-fbo) iff $f^{c}$ is $(r, s)$-fbc set.

The collection of all $(r, s)$-fbc sets is denoted by $b \mathcal{C}_{\tau, r, s}$ and that of $(r, s)$ fbo sets is denoted by $b \mathcal{O}_{\tau, r, s}$.

Mohammed et. al.[4] introduced the concepts $(r, s)$-generalized fuzzy $b$-closed sets and $(r, s)$-generalized fuzzy $b$-open sets in terms of the double fuzzy $b$-closure and double fuzzy $b$-interior operators as defined below:

Definition 2.9. [4] Let $\left(X, \tau, \tau^{*}\right)$ be a dfts. Then double fuzzy b-closure operator and double fuzzy $b$-interior operator are defined by $b C_{\tau, \tau^{*}}(f, r, s)=\wedge\left\{h \in I^{X}: f \leq h\right.$ and $\left.h \in b \mathcal{C}_{\tau, r, s}\right\}$, $b I_{\tau, \tau^{*}}(f, r, s)=\vee\left\{h \in I^{X}: h \leq f\right.$ and $\left.h \in b \mathcal{O}_{\tau, r, s}\right\}$

where $(r, s) \in I_{0} \oplus I_{1}$. 
Definition 2.10. [4] Let $\left(X, \tau, \tau^{*}\right)$ be a dfts, $f \in I^{X},(r, s) \in I_{0} \oplus I_{1}$, then $f$ is called

1. $(r, s)$-generalized fuzzy $b$-closed (or, $(r, s)$-gfbc) set if $b C_{\tau, \tau^{*}}(f, r, s) \leq$ $h$ whenever $f \leq h$ and $h \in \mathcal{O}_{\tau, r, s}$.

2. $(r, s)$-generalized fuzzy b-open (or, $(r, s)$-gfbo) set if $f^{c}$ is a $(r, s)$-gfbc set.

We denote the collection of all $(r, s)$-gfbc sets by $G b \mathcal{C}_{\tau, r, s}$ and the collection of all $(r, s)$-gfbo sets by $G b \mathcal{O}_{\tau, r, s}$.

Definition 2.11. [8] Let $\left(X, \tau, \tau^{*}\right)$ be a dfts and $g \in I^{X}$. For $\alpha \in I_{0}$ and $\beta \in I_{1}$ with $\alpha \geq \tau(g), \beta \leq \tau^{*}(g)$ and $\alpha+\beta \leq 1$, define $\tau_{0}, \tau_{0}^{*}: I^{X} \rightarrow I$ by

(i) $\tau_{0}(g)=\alpha, \tau_{0}^{*}(g)=\beta$ and

(ii) for all $f \in I^{X} \backslash\{g\}$,

$$
\begin{aligned}
& \tau_{0}(f)=\max \left\{\tau(f), \bigvee\left\{\tau\left(f_{1}\right) \wedge \tau\left(f_{2}\right) \wedge \alpha:\left(f_{1}, f_{2}\right) \in R_{g} f\right\}\right\} \\
& \tau_{0}^{*}(f)=\min \left\{\tau^{*}(f), \bigwedge\left\{\tau^{*}\left(f_{1}\right) \vee \tau^{*}\left(f_{2}\right) \vee \beta:\left(f_{1}, f_{2}\right) \in R_{g} f\right\}\right\}
\end{aligned}
$$

where $R_{g} f=\left\{\left(f_{1}, f_{2}\right): f=f_{1} \vee\left(f_{2} \wedge g\right) ; f_{1}, f_{2} \in I^{X}\right\}$

Then the triplet $\left(X, \tau_{0}, \tau_{0}^{*}\right)$ is a dfts called the $(g, \alpha, \beta)$-extension of $\left(X, \tau, \tau^{*}\right)$.

\section{Regularity and extensions}

This section answers the question, "whether the extensions of a regular double fuzzy topological space remain regular or not?".

The regularity axiom in a double fuzzy topological space is defined as follows:

Definition 3.1. A dfts $\left(X, \tau, \tau^{*}\right)$ is said to be regular if for any fuzzy point $x_{\lambda}$ in $X$ and any $f \in \mathcal{O}_{\tau, r, s}$ with $x_{\lambda} \in f$, there exists $h \in \mathcal{O}_{\tau, r, s}$ such that $x_{\lambda} \in h \leq C_{\tau, \tau^{*}}(h, r, s) \leq f$.

In general, the regularity of a dfts is not carried over to its extensions as shown below: 
Example 3.2. Let $X=\{x, y\}$ and $\tau, \tau^{*}$ be as follows:

$$
\tau(f)= \begin{cases}1, & \text { if } f \in\{\underline{0}, \underline{1}\} \\ \frac{1}{4}, & \text { if } f \in\left\{x_{\frac{1}{2}}, x_{1}, y_{1}, x_{\frac{1}{2}} \vee y_{1}\right\} \\ 0, & \text { otherwise. }\end{cases}
$$

and

$$
\tau^{*}(f)= \begin{cases}0, & \text { if } f \in\{\underline{0}, \underline{1}\} \\ \frac{1}{3}, & \text { if } f \in\left\{x_{\frac{1}{2}}, x_{1}, y_{1}, x_{\frac{1}{2}} \vee y_{1}\right\} \\ 1, & \text { otherwise. }\end{cases}
$$

Then, $\left(X, \tau, \tau^{*}\right)$ is a regular dfts.

Let $\left(X, \tau_{0}, \tau_{0}^{*}\right)$ be the $\left(g, \frac{1}{4}, \frac{1}{3}\right)$-extension of $\left(X, \tau, \tau^{*}\right)$ with $g=y_{\frac{1}{4}}$. Then, the resulting double fuzzy topology $\left(\tau_{0}, \tau_{0}^{*}\right)$ on $X$ is given by,

$$
\tau_{0}(f)= \begin{cases}1, & \text { if } f \in\{\underline{0}, \underline{1}\} \\ \frac{1}{4}, & \text { if } f \in\left\{x_{\frac{1}{2}}, x_{1}, y_{1},, x_{\frac{1}{2}} \vee y_{1}, y_{\frac{1}{4}}, x_{1} \vee y_{\frac{1}{4}}, x_{\frac{1}{2}} \vee y_{\frac{1}{4}}\right\} \\ 0, & \text { elsewhere }\end{cases}
$$

and

$$
\tau_{0}^{*}(f)= \begin{cases}0, & \text { if } f \in\{\underline{0}, \underline{1}\} \\ \frac{1}{3}, & \text { if } f \in\left\{x_{\frac{1}{2}}, x_{1}, y_{1},, x_{\frac{1}{2}} \vee y_{1}, y_{\frac{1}{4}}, x_{1} \vee y_{\frac{1}{4}}, x_{\frac{1}{2}} \vee y_{\frac{1}{4}}\right\} \\ 1, & \text { elsewhere. }\end{cases}
$$

But $\left(X, \tau_{0}, \tau_{0}^{*}\right)$ is not regular since for $(r, s)=\left(\frac{1}{4}, \frac{1}{3}\right), x_{\lambda}=y_{\frac{1}{4}}$ and $f=x_{\frac{1}{2}} \vee y_{\frac{1}{4}} \in \mathcal{O}_{\tau, r, s}$ with $x_{\lambda} \in f$ there does not exist $h \in \mathcal{O}_{\tau, r, s}$ such that $x_{\lambda} \in h \leq C_{\tau, \tau^{*}}(h, r, s) \leq f$.

Lemma 3.3. Let $\left(X, \tau_{0}, \tau_{0}^{*}\right)$ be the $(g, \alpha, \beta)$-extension of a dfts $\left(X, \tau, \tau^{*}\right)$ with $\tau(f)+\tau^{*}(f)=1, \forall f \in I^{X}$ and $R(\tau)$, the range of $\tau$, is finite. Let $(r, s) \in I_{0} \oplus I_{1}$ and $f \in \mathcal{O}_{\tau_{0}, r, s}$. Then there exist $f_{1}, f_{2} \in \mathcal{O}_{\tau, r, s}$ such that $f=f_{1} \vee\left(f_{2} \wedge g\right)$.

Since $\tau(f)+\tau^{*}(f)=1$ and $R(\tau)$ is finite, $R\left(\tau^{*}\right)$ is finite.

Let $f \in \mathcal{O}_{\tau_{0}, r, s}$. Then, from the definition of $(g, \alpha, \beta)$-extension, we have either $f \in \mathcal{O}_{\tau, r, s}$ or $\tau(f) \geq r$ and $\bigwedge\left\{\tau^{*}\left(f_{1}\right) \vee \tau^{*}\left(f_{2}\right) \vee \beta:\left(f_{1}, f_{2}\right) \in R_{g} f\right\} \leq s$ or $\bigvee\left\{\tau\left(f_{1}\right) \wedge \tau\left(f_{2}\right) \wedge \alpha:\left(f_{1}, f_{2}\right) \in R_{g} f\right\} \geq r$ and $\tau^{*}(f) \leq s$ or $\bigvee\left\{\tau\left(f_{1}\right) \wedge\right.$ 
$\left.\tau\left(f_{2}\right) \wedge \alpha:\left(f_{1}, f_{2}\right) \in R_{g} f\right\} \geq r$ and $\bigwedge\left\{\tau^{*}\left(f_{1}\right) \vee \tau^{*}\left(f_{2}\right) \vee \beta:\left(f_{1}, f_{2}\right) \in R_{g} f\right\} \leq$

Case 1: $f \in \mathcal{O}_{\tau, r, s}$

Then, there exist $f, \underline{0} \in \mathcal{O}_{\tau, r, s}$ such that $(f, \underline{0}) \in R_{g} f$.

Case 2: $\tau(f) \geq r$ and $\bigwedge\left\{\tau^{*}\left(f_{1}\right) \vee \tau^{*}\left(f_{2}\right) \vee \beta:\left(f_{1}, f_{2}\right) \in R_{g} f\right\} \leq s$ $\bigwedge\left\{\tau^{*}\left(f_{1}\right) \vee \tau^{*}\left(f_{2}\right) \vee \beta:\left(f_{1}, f_{2}\right) \in R_{g} f\right\} \leq s$

$\Longrightarrow \exists\left(f_{1}, f_{2}\right) \in R_{g} f$ such that $\tau^{*}\left(f_{1}\right) \vee \tau^{*}\left(f_{2}\right) \vee \beta \leq s$, since $R\left(\tau^{*}\right)$ is finite

$\Longrightarrow \exists\left(f_{1}, f_{2}\right) \in R_{g} f$ such that $\tau^{*}\left(f_{1}\right) \vee \tau^{*}\left(f_{2}\right) \leq s$

$\Longrightarrow \exists\left(f_{1}, f_{2}\right) \in R_{g} f$ such that

$$
\tau\left(f_{1}\right) \wedge \tau\left(f_{2}\right)=1-\left(\tau^{*}\left(f_{1}\right) \vee \tau^{*}\left(f_{2}\right)\right) \geq 1-s \geq r .
$$

Thus, there exist $f_{1}, f_{2} \in \mathcal{O}_{\tau, r, s}$ such that $f=f_{1} \vee\left(f_{2} \wedge g\right)$.

Case 3: $\bigvee\left\{\tau\left(f_{1}\right) \wedge \tau\left(f_{2}\right) \wedge \alpha:\left(f_{1}, f_{2}\right) \in R_{g} f\right\} \geq r$ and $\tau^{*}(f) \leq s$ $\left.\bigvee\left\{\tau f_{1}\right) \wedge \tau\left(f_{2}\right) \vee \alpha:\left(f_{1}, f_{2}\right) \in R_{g} f\right\} \geq r$

$\Longrightarrow \exists\left(f_{1}, f_{2}\right) \in R_{g} f$ such that $\tau\left(f_{1}\right) \wedge \tau\left(f_{2}\right) \wedge \alpha \geq r$, since $R(\tau)$ is finite

$\Longrightarrow \exists\left(f_{1}, f_{2}\right) \in R_{g} f$ such that $\tau\left(f_{1}\right) \wedge \tau\left(f_{2}\right) \geq r$

$\Longrightarrow \exists\left(f_{1}, f_{2}\right) \in R_{g} f$ such that

$$
\tau^{*}\left(f_{1}\right) \vee \tau^{*}\left(f_{2}\right)=1-\left(\tau\left(f_{1}\right) \wedge \tau\left(f_{2}\right)\right) \leq 1-r \leq s .
$$

i.e., there exist $f_{1}, f_{2} \in \mathcal{O}_{\tau, r, s}$ such that $f=f_{1} \vee\left(f_{2} \wedge g\right)$.

Case 4: $\bigvee\left\{\tau\left(f_{1}\right) \wedge \tau\left(f_{2}\right) \wedge \alpha:\left(f_{1}, f_{2}\right) \in R_{g} f\right\} \geq r$ and $\bigwedge\left\{\tau^{*}\left(f_{1}\right) \vee \tau^{*}\left(f_{2}\right) \vee \beta:\left(f_{1}, f_{2}\right) \in R_{g} f\right\} \leq s$

Proceeding as in Case 3 we get $f_{1}, f_{2} \in \mathcal{O}_{\tau, r, s}$ such that $f=f_{1} \vee\left(f_{2} \wedge g\right)$.

Now, the following theorem gives a sufficient condition for the extension to be regular.

Theorem 3.4. Let $\left(X, \tau, \tau^{*}\right)$ be a regular dfts such that $\tau(f)+\tau^{*}(f)=1$, $\forall f \in I^{X}$ and $R(\tau)$ is finite. Then the $(g, 1,0)$-extension of $\left(X, \tau, \tau^{*}\right)$ is regular, provided $g \in \mathcal{C}_{\tau, 1,0}$.

Let $\left(X, \tau_{0}, \tau_{0}^{*}\right)$ be the $(g, 1,0)$-extension of $\left(X, \tau, \tau^{*}\right)$ where $g \in \mathcal{C}_{\tau, 1,0}$ and let $f \in \mathcal{O}_{\tau_{0}, r, s}$. Then by lemma 3.3, there exist $f_{1}, f_{2} \in \mathcal{O}_{\tau, r, s}$ such that $f=f_{1} \vee\left(f_{2} \wedge g\right)$.

Now for a fuzzy point $x_{\lambda}$,

$$
\begin{aligned}
x_{\lambda} \in f & \Longrightarrow x_{\lambda} \in f_{1} \vee\left(f_{2} \wedge g\right) \\
& \Longrightarrow \text { either } x_{\lambda} \in f_{1} \text { or } x_{\lambda} \in f_{2} \wedge g
\end{aligned}
$$


If $x_{\lambda} \in f_{1}$, then $\exists h \in \mathcal{O}_{\tau, r, s}$ such that $x_{\lambda} \in h \leq C_{\tau, \tau^{*}}(h, r, s) \leq f_{1}$. Clearly, $\exists h \in \mathcal{O}_{\tau_{0}, r, s}$ such that $x_{\lambda} \in h \leq C_{\tau_{0}, \tau_{0}^{*}}(h, r, s) \leq f_{1} \leq f$.

Further, $x_{\lambda} \in f_{2} \wedge g \Longrightarrow x_{\lambda} \in f_{2}$ and $x_{\lambda} \in g$. Then, $\exists h^{*} \in \mathcal{O}_{\tau, r, s}$ such that $x_{\lambda} \in h^{*} \leq C_{\tau, \tau^{*}}\left(h^{*}, r, s\right) \leq f_{2}$ so that $x_{\lambda} \in h^{*} \leq C_{\tau_{0}, \tau_{0}^{*}}\left(h^{*}, r, s\right) \leq f_{2}$.

Hence, $x_{\lambda} \in h^{*} \wedge g \leq C_{\tau_{0}, \tau_{0}^{*}}\left(h^{*} \wedge g, r, s\right) \leq f_{2} \wedge g$ and $\tau_{0}\left(h^{*} \wedge g\right) \geq \tau_{0}\left(h^{*}\right) \wedge$ $\tau_{0}(g) \geq r$. Similarly, $\tau_{0}^{*}\left(h^{*} \wedge g\right) \leq s$. Thus, $h^{*} \wedge g \in \mathcal{O}_{\tau_{0}, r, s}$.

Remark 3.5. The converse of the above theorem is not true in general. For example, let $X=\{y, z\}$ and $\tau, \tau^{*}: I^{X} \rightarrow I$ be as follows:

$$
\tau(f)= \begin{cases}1, & \text { if } f \in\left\{\underline{0}, \underline{1}, y_{1}\right\} \\ \frac{9}{20}, & \text { if } f=y_{\frac{1}{2}} \\ 0, & \text { otherwise. }\end{cases}
$$

and

$$
\tau^{*}(f)= \begin{cases}0, & \text { if } f \in\left\{\underline{0}, \underline{1}, y_{1}\right\} \\ \frac{11}{20}, & \text { if } f=y_{1} \\ 1, & \text { otherwise. }\end{cases}
$$

Then, $\left(X, \tau, \tau^{*}\right)$ is not a regular dfts since for any $(r, s) \leq\left(\frac{9}{20}, \frac{11}{20}\right), y_{1} \in$ $\mathcal{O}_{\tau, r, s}$ and the fuzzy point $y_{\frac{1}{2}} \in y_{1}$, but not exist $h \in \mathcal{O}_{\tau, r, s}$ such that $y_{\frac{1}{2}} \in h \leq C_{\tau, \tau^{*}}(h, r, s) \leq y_{1}$. Also, note that $\tau(f)+\tau^{*}(f)=1, \forall f \in I^{X}$.

Now, consider the $(g, 1,0)$ extension $\left(X, \tau_{0}, \tau_{0}^{*}\right)$ of $\left(X, \tau, \tau^{*}\right)$, where $g=$ $z_{1} \in \mathcal{C}_{\tau, 1,0}$. Then $\tau_{0}$ and $\tau_{0}^{*}$ are given by

$$
\tau_{0}(f)= \begin{cases}1, & \text { if } f \in\left\{\underline{0}, \underline{1}, z_{1}, y_{1}\right\} \\ \frac{9}{20}, & \text { if } f \in\left\{y_{\frac{1}{2}}, y_{\frac{1}{2}} \vee z_{1}\right\} \\ 0, & \text { otherwise. }\end{cases}
$$

and

$$
\tau_{0}^{*}(f)= \begin{cases}0, & \text { if } f \in\left\{\underline{0}, \underline{1}, z_{1}, y_{1}\right\} \\ \frac{11}{20}, & \text { if } f \in\left\{y_{\frac{1}{2}}, y_{\frac{1}{2}} \vee z_{1}\right\} \\ 1, & \text { otherwise }\end{cases}
$$

Clearly, $\left(X, \tau_{0}, \tau_{0}^{*}\right)$ is a regular dfts since for any fuzzy point $x_{\lambda}$ in $X$ and any $f \in \mathcal{O}_{\tau, r, s},(r, s) \in I_{0} \oplus I_{1}$ with $x_{\lambda} \in f$, by taking $h=f$, the condition for regularity is satisfied. 
The conditions $\tau(f)+\tau^{*}(f)=1$ and $g \in \mathcal{C}_{\tau, 1,0}$ in Theorem 3.4 is not necessary as verified below:

Example 3.6. Let $X=I$ and define a double fuzzy topology $\left(\tau, \tau^{*}\right)$ on $X$ as follows:

$$
\tau(f)= \begin{cases}1, & \text { if } f \in\{\underline{0}, \underline{1}\} \\ \frac{9}{20}, & \text { if } f \in\left\{\left(\frac{1}{4}\right)_{\frac{1}{3}},\left(\frac{7}{25}\right)_{\frac{1}{3}},\left(\frac{1}{4}\right)_{\frac{1}{3}}^{c},\left(\frac{7}{25}\right)_{\frac{1}{3}}^{c}\right\} \\ 0, & \text { otherwise. }\end{cases}
$$

and

$$
\tau^{*}(f)= \begin{cases}0, & \text { if } f \in\{\underline{0}, \underline{1}\} \\ \frac{2}{5}, & \text { if } f \in\left\{\left(\frac{1}{4}\right)_{\frac{1}{3}},\left(\frac{7}{25}\right)_{\frac{1}{3}},\left(\frac{1}{4}\right)_{\frac{1}{3}}^{c},\left(\frac{7}{25}\right)_{\frac{1}{3}}^{c}\right\} \\ 1, & \text { otherwise. }\end{cases}
$$

Clearly, $\left(X, \tau, \tau^{*}\right)$ is a regular dfts.

Let $\left(X, \tau_{0}, \tau_{0}^{*}\right)$ be the $(g, 1,0)$-extension of $\left(X, \tau, \tau^{*}\right)$ where $g=\left(\frac{1}{2}\right) \notin$ $\mathcal{C}_{\tau, 1,0}$. Then, $\tau_{0}$ and $\tau_{0}^{*}$ are given by

$$
\tau_{0}(f)= \begin{cases}1, & \text { if } f \in\left\{\underline{0}, \underline{1},\left(\frac{1}{2}\right)\right\} \\ \frac{9}{20}, & \text { if } f \in\left\{\left(\frac{1}{4}\right)_{\frac{1}{3}},\left(\frac{7}{25}\right)_{\frac{1}{3}},\left(\frac{1}{4}\right)_{\frac{1}{3}}^{c},\left(\frac{7}{25}\right)_{\frac{1}{3}}^{c}\right\} \\ 0, & \text { otherwise. }\end{cases}
$$

and

$$
\tau_{0}^{*}(f)= \begin{cases}0, & \text { if } f \in\left\{\underline{0}, \underline{1},\left(\frac{1}{2}\right)\right\} \\ \frac{2}{5}, & \text { if } f \in\left\{\left(\frac{1}{4}\right)_{\frac{1}{3}},\left(\frac{7}{25}\right)_{\frac{1}{3}},\left(\frac{1}{4}\right)_{\frac{1}{3}}^{c},\left(\frac{7}{25}\right)_{\frac{1}{3}}^{c}\right\} \\ 1, & \text { otherwise. }\end{cases}
$$

Now, for any fuzzy point $x_{\lambda}$ and $f \in \mathcal{O}_{\tau, r, s},(r, s) \in I_{0} \oplus I_{1}$ with $x_{\lambda} \in f$, there exists $h=f$ such that $x_{\lambda} \in h \leq C_{\tau_{0}, \tau_{0}^{*}}(h, r, s) \leq f$. Hence, $\left(X, \tau_{0}, \tau_{0}^{*}\right)$ is regular.

Remark 3.7. Consider the double fuzzy topological space $\left(X, \tau, \tau^{*}\right)$ and its extension $\left(X, \tau_{0}, \tau_{0}^{*}\right)$ as defined in Remark 3.5. Then it follows that the regularity of the extension does not guarantee the regularity of the original space. 


\section{Closed sets and extensions}

In [10], it is shown that the partial ordering in $I_{0} \oplus I_{1}$ induces an ordering in the collection of all $\mathcal{C}_{\tau, r, s}$. However, the same partial ordering does not induce an order for $G \mathcal{C}_{\tau, r, s}, R G \mathcal{C}_{\tau, r, s}, b \mathcal{C}_{\tau, r, s}$ and $G b \mathcal{C}_{\tau, r, s}$ as shown below:

Example 4.1. Let $X=\{a, b, c\}$, and define a double fuzzy topology on $X$ as follows:

$$
\tau(f)= \begin{cases}1, & \text { if } f \in\{\underline{0}, \underline{1}\} \\ 1-\alpha, & \text { if } f=\underline{\alpha}, \alpha \in I \backslash\{0,1\} \\ 0, & \text { otherwise. }\end{cases}
$$

and

$$
\tau^{*}(f)= \begin{cases}0, & \text { if } f \in\{\underline{0}, \underline{1}\} \\ \alpha, & \text { if } f=\underline{\alpha}, \alpha \in I \backslash\{0,1\} \\ 1, & \text { otherwise. }\end{cases}
$$

Now, for $\left(r_{1}, s_{1}\right)=\left(\frac{2}{3}, \frac{1}{3}\right), C_{\tau, \tau^{*}}\left(\left(\frac{2}{5}\right), r_{1}, s_{1}\right)=\left(\underline{\frac{2}{3}}\right)$ and hence $\left(\underline{\frac{2}{5}}\right) \in$ $G \mathcal{C}_{\tau, r_{1}, s_{1}}$ since the only $f \in \mathcal{O}_{\tau, r_{1}, s_{1}}$ such that $\left(\frac{2}{5}\right) \leq f$ is $f=\underline{1}$.

Again, for $\left(r_{2}, s_{2}\right)=\left(\frac{3}{5}, \frac{1}{3}\right), C_{\tau, \tau^{*}}\left(\left(\frac{2}{5}\right), r_{2}, s_{2}\right)=\left(\frac{3}{5}\right)$ and hence $\left(\underline{\frac{2}{5}}\right) \notin$ $G \mathcal{C}_{\tau, r_{2}, s_{2}}$ since $C_{\tau, \tau^{*}}\left(\left(\frac{2}{5}\right), r_{2}, s_{2}\right) \not{Z}\left(\frac{2}{5}\right)$ where $\left(\frac{2}{5}\right) \in \mathcal{O}_{\tau, r_{2}, s_{2}}$.

i.e., $G \mathcal{C}_{\tau, r_{1}, s_{1}} \nsubseteq \overline{G \mathcal{C}}_{\tau, r_{2}, s_{2}}$ for $\left(r_{1}, s_{1}\right),\left(r_{2}, s_{2}\right) \in I_{0} \oplus I_{1}$ with $\left(r_{2}, s_{2}\right) \leq$ $\left(r_{1}, s_{1}\right)$.

Again, $R \mathcal{O}_{\tau, r_{1}, s_{1}}=\left\{\underline{0}, \underline{1},\left(\underline{\frac{1}{3}}\right)\right\}$ and $\left(\frac{2}{5}\right) \in R G \mathcal{C}_{\tau, r_{1}, s_{1}}$. But, $\left(\underline{\frac{2}{5}}\right) \notin R G \mathcal{C}_{\tau, r_{2}, s_{2}}$ since $\left(\frac{2}{5}\right) \in R \mathcal{O}_{\tau, r_{2}, s_{2}}$ and $C_{\tau, \tau^{*}}\left(\left(\frac{2}{5}\right), r_{2}, s_{2}\right)=\left(\frac{3}{5}\right) \not \leq\left(\frac{2}{5}\right)$.

Further, $\left(\underline{\frac{1}{3}}\right) \in b \mathcal{C}_{\tau, r_{1}, s_{1}}$. But, $\left(\underline{\frac{1}{3}}\right) \notin b \mathcal{C}_{\tau, r_{2}, s_{2}}$ since $C_{\tau, \tau^{*}}\left(\left(\underline{\frac{1}{3}}\right), r_{2}, s_{2}\right)=$ $\left(\underline{\frac{3}{5}}\right)$ and $I_{\tau, \tau^{*}}\left(C_{\tau, \tau^{*}}\left(\left(\underline{\frac{1}{3}}\right), r_{2}, s_{2}\right), r_{2}, s_{2}\right)=\left(\underline{\frac{2}{5}}\right)$.

However, we have the following theorem establishing inclusions among certain families of closed in a double fuzzy topological space.

Theorem 4.2. Let $\left(X, \tau, \tau^{*}\right)$ be a dfts. Then, for any $\left(r_{1}, s_{1}\right),\left(r_{2}, s_{2}\right) \in$ $I_{0} \oplus I_{1}$ with $\left(r_{2}, s_{2}\right) \leq\left(r_{1}, s_{1}\right)$,

1. $\mathcal{C}_{\tau, r_{1}, s_{1}} \subseteq G \mathcal{C}_{\tau, r_{2}, s_{2}} \subseteq R G \mathcal{C}_{\tau, r_{2}, s_{2}}$

2. $\mathcal{C}_{\tau, r_{1}, s_{1}} \subseteq b \mathcal{C}_{\tau, r_{2}, s_{2}} \subseteq G b \mathcal{C}_{\tau, r_{2}, s_{2}}$

Note that in general, the families $G \mathcal{C}_{\tau, r, s}, R G \mathcal{C}_{\tau, r, s}, b \mathcal{C}_{\tau, r, s}$ and $G b \mathcal{C}_{\tau, r, s}$ do not remain intact while taking extensions. 
Example 4.3. Consider the dfts $\left(X, \tau, \tau^{*}\right)$ defined in Example 4.1 and the fuzzy set $g$ defined by

$$
g(x)= \begin{cases}\frac{1}{2}, & \text { if } x=\text { aor } x=b \\ \frac{9}{20}, & \text { if } x=c\end{cases}
$$

Let $\left(X, \tau_{0}, \tau_{0}^{*}\right)$ be the $\left(g, \frac{2}{3}, \frac{1}{3}\right)$-extension of $\left(X, \tau, \tau^{*}\right)$. Then, for $\left(r_{1}, s_{1}\right)=$ $\left(\frac{2}{3}, \frac{1}{3}\right)$ we have $(\underline{2}) \in G \mathcal{C}_{\tau, r_{1}, s_{1}}$. But, $\left(\underline{\frac{2}{5}}\right) \notin G \mathcal{C}_{\tau_{0}, r_{1}, s_{1}}$ since $(\underline{2}) \leq g \in$ $\mathcal{O}_{\tau_{0}, r_{1}, s_{1}}$ but $C_{\tau_{0}, \tau_{0}^{*}}\left(\left(\frac{2}{5}\right), r_{1}, s_{1}\right)=g^{c} \not \leq g$. Hence, $G \mathcal{C}_{\tau, r_{1}, s_{1}} \not \subset G \mathcal{C}_{\tau_{0}, r_{1}, s_{1}}$.

Now, $g \in R \mathcal{O}_{\tau_{0}, r_{1}, s_{1}}$ since $I_{\tau_{0}, \tau_{0}^{*}}\left(C_{\tau_{0}, \tau_{0}^{*}}\left(g, r_{1}, s_{1}\right), r_{1}, s_{1}\right)=I_{\tau_{0}, \tau_{0}^{*}}\left(g^{c}, r_{1}, s_{1}\right)=$ $g$. Then, $\left(\frac{2}{5}\right) \leq g$ but $C_{\tau_{0}, \tau_{0}^{*}}\left(\left(\frac{2}{5}\right), r_{1}, s_{1}\right) \not \leq g$ which shows that $R G \mathcal{C}_{\tau, r_{1}, s_{1}} \nsubseteq$ $R G \mathcal{C}_{\tau_{0}, r_{1}, s_{1}}$ since $\left(\underline{\frac{2}{5}}\right) \in R G \mathcal{C}_{\tau, r_{1}, s_{1}}$.

Again, $\left(\underline{\frac{1}{3}}\right) \in b \overline{\mathcal{C}}_{\tau, r_{1}, s_{1}}$. Also, $I_{\tau_{0}, \tau_{0}^{*}}\left(C_{\tau_{0}, \tau_{0}^{*}}\left(\left(\underline{\frac{1}{3}}\right), r_{1}, s_{1}\right), r_{1}, s_{1}\right)=I_{\tau_{0}, \tau_{0}^{*}}\left(g^{c}, r_{1}, s_{1}\right)=$ $g$ and $C_{\tau_{0}, \tau_{0}^{*}}\left(I_{\tau_{0}, \tau_{0}^{*}}\left(\left(\underline{\frac{1}{3}}\right), r_{1}, s_{1}\right), r_{1}, s_{1}\right)=C_{\tau_{0}, \tau_{0}^{*}}\left(\left(\underline{\frac{1}{3}}\right), r_{1}, s_{1}\right)=g^{c}$.

i.e, $\left(\underline{\frac{1}{3}}\right) \notin b \mathcal{C}_{\tau_{0}, r_{1}, s_{1}}$.

Further, $\left(\underline{\frac{1}{3}}\right) \in G b \mathcal{C}_{\tau, r_{1}, s_{1}}$ and $b \mathcal{C}_{\tau_{0}, r_{1}, s_{1}}=\left\{f \in I^{X}: g \leq f \leq \underline{1}\right\} \vee\{\underline{0}\}$. Then, $b C_{\tau_{0}, \tau_{0}^{*}}\left(\left(\underline{\frac{1}{3}}\right), r_{1}, s_{1}\right)=g$ and therefore $\left(\underline{\frac{1}{3}}\right) \notin G b \mathcal{C}_{\tau_{0}, r_{1}, s_{1}}$ since $\left(\underline{\frac{1}{3}}\right) \in$ $\mathcal{O}_{\tau_{0}, r_{1}, s_{1}}$ but $b C_{\tau_{0}, \tau_{0}^{*}}\left(\left(\underline{\frac{1}{3}}\right), r_{1}, s_{1}\right) \not \leq\left(\frac{1}{3}\right)$.

i.e., $G b \mathcal{C}_{\tau, r_{1}, s_{1}} \nsubseteq G b \mathcal{C}_{\tau_{0}, r_{1}, s_{1}}$.

However, the following theorem identifies some families that remain intact under extensions.

Theorem 4.4. Let $\left(X, \tau, \tau^{*}\right)$ be a dfts and $\left(X, \tau_{0}, \tau_{0}^{*}\right)$ be its $(g, \alpha, \beta)$ extension. Then for any $(r, s) \in I_{0} \oplus I_{1}$ such that $(\alpha, \beta) \leq(r, s), G \mathcal{C}_{\tau, r, s}=$ $G \mathcal{C}_{\tau_{0}, r, s}, R G \mathcal{C}_{\tau, r, s}=R G \mathcal{C}_{\tau_{0}, r, s}, b \mathcal{C}_{\tau, r, s}=b \mathcal{C}_{\tau_{0}, r, s}$ and $G b \mathcal{C}_{\tau, r, s}=G b \mathcal{C}_{\tau_{0}, r, s}$.

Since $\tau\left(f_{1}\right) \wedge \tau\left(f_{2}\right) \wedge \alpha \leq \alpha<r, \tau^{*}\left(f_{1}\right) \vee \tau^{*}\left(f_{2}\right) \vee \beta \geq \beta>s$ for all $f_{1}, f_{2} \in I^{X}$, we have $\mathcal{O}_{\tau, r, s}=\mathcal{O}_{\tau_{0}, r, s}$. Therefore, $\mathcal{C}_{\tau, r, s}=\mathcal{C}_{\tau_{0}, r, s}$.

Hence, $I_{\tau_{0}, \tau_{0}^{*}}(f, r, s)=I_{\tau, \tau^{*}}(f, r, s)$ and $C_{\tau_{0}, \tau_{0}^{*}}(f, r, s)=C_{\tau, \tau^{*}}(f, r, s)$, for all $f \in I^{X}$.

Again, $C_{\tau_{0}, \tau_{0}^{*}}\left(I_{\tau_{0}, \tau_{0}^{*}}(f, r, s), r, s\right)=C_{\tau, \tau^{*}}\left(I_{\tau, \tau^{*}}(f, r, s), r, s\right)$ and $I_{\tau_{0}, \tau_{0}^{*}}\left(C_{\tau_{0}, \tau_{0}^{*}}(f, r, s), r, s\right)=I_{\tau, \tau^{*}}\left(C_{\tau, \tau^{*}}(f, r, s), r, s\right)$. Hence the proof. 


\section{Conclusion}

Looking into the regularity of a given double fuzzy topological space and its extensions, it is found that the regularity of the given space does not guarantee regularity of the extended space. However, certain situations under which the regularity of a double fuzzy topological space is carried over to its extensions are obtained. Further, some types of extensions which keep the families of certain types of closed sets intact are identified.

\section{References}

[1] S. E. Abbas, "(R,S)-generalized intuitionistic fuzzy closed sets”, Journal of the egyptian mathematical society, vol. 14,no. 2, pp. 331-351, 2006

[2] A. Ghareeb, "Normality of double fuzzy topological spaces", Applied mathematics letters, vol. 24 , no. 4, pp. 533-540, 2011. doi: 10.1016/ j.aml.2010.11.008

[3] E. P. Lee and Y. B. Im, "Mated fuzzy topological spaces", Journal of the Korean institute of intelligent systems, vol. 11, no. 2, pp. 161-165, 2001.

[4] F. M. Mohammed, M. S. M. Noorani, and A. Ghareeb, "Generalized fuzzy B-closed and generalized $\square$-fuzzy B-closed sets in double fuzzy topological spaces", Egyptian journal of basic and applied sciences, vol. 3, no. 1, pp. 61-67, 2016. doi: 10.1016/j.ejbas.2015.09.001

[5] T. K. Mondal and S. K. Samanta, "On intuitionistic gradation of openness", Fuzzy sets and systems, vol. 131, no. 3, pp. 323-336, 2002. doi: 10.1016/ s0165-0114(01)00235-4

[6] A. A. Ramadan, S. E. Abbas, and A. A. Abd El-Latif, "Compactness in intuitionistic fuzzy topological spaces", International journal of mathematics and mathematical sciences, vol. 2005, no. 1, pp. 19-32, 2005. doi: 10.1155/ijmms.2005.19

[7] G. C. Ray and B. C. Tripathy, "Fuzzy $\delta *$-almost continuous and fuzzy $\delta *$-continuous functions in mixed fuzzy ideal topological spaces", Proyecciones (Antofagasta), vol. 39, no. 2, pp. 435-449, 2020. doi: 10.22199/issn.0717-6279-2020-02-0027

[8] S. Vivek and S. C. Mathew, "On the extensions of a double fuzzy topological space", Journal of advanced studies in topology, vol. 9, no. 1, pp. 75-93, 2018. doi: 10.20454/ jast.2018.1431 
[9] S. Vivek and S. C. Mathew, "On regular fuzzy closed sets in a double fuzzy topological space", Advances in fuzzy sets and systems, vol. 24, no. 2, pp. 55-73, 2019. doi: 10.17654/ fs024020055

[10] S. Vivek and S. C. Mathew, "Some lattices associated with a double fuzzy topological spaces", Journal of fuzzy mathematics, vol. 27, no. 1, pp. 153-170, 2019

[11] S. Vivek and S. C. Mathew, "Normality of the extensions of a double fuzzy topological space", Advances in mathematics: scientific journal, vol. 9, no. 11, pp. 9455-9462, 2020. doi: 10.37418/ amsj.9.11.49

[12] S. Vivek and S. C. Mathew, "Regular fuzzy closed sets and extensions of a double fuzzy topological space”, Journal of mathematical extension, vol. 14, no. 4, pp. 101-112, 2020.

[13] B. C. Tripathy and G. C. Ray, "On mixed fuzzy topological spaces and countability”, Soft computing, vol. 16, no. 10, pp. 1691-1695, 2012. doi: 10.1007/ s00500-012-0853-1

[14] B. C. Tripathy and G. C. Ray, "Mixed fuzzy ideal topological spaces", Applied mathematics and computation, vol. 220, pp. 602-607, 2013. doi: 10.1016/j.amc.2013.05.072

[15] B. C. Tripathy and G. C. Ray, "On ס-continuity in mixed fuzzy topological spaces", Boletim da Sociedade paranaense de matemática, vol. 32, no. 2, pp. 175-187, 2014. doi: 10.5269/ bspm.v32i2.20254

[16] B. C. Tripathy and G. C. Ray, "Weakly continuous functions on mixed fuzzy topological spaces", Acta scientiarum. technology, vol. 36, no. 2, p. 331, 2014. doi: 10.4025/ actascitechnol.v36i2.16241 


\section{Vivek S.}

Department of Mathematics,

St. Thomas College, Palai

Arunapuram P. O. 686574,

Kottayam, Kerala,

India

e-mail: vivekmaikkattu@yahoo.com

and

\section{Sunil C. Mathew}

Department of Mathematics,

Deva Matha College Kuravilangad,

Kuravilangad P. O. 686633,

Kottayam, Kerala,

India

e-mail: sunilcmathew@gmail.com,

Corresponding Author 